COMMENT. This is the first report of a familial multigenerational case of Glut-1 deficiency syndrome. De Vivo et al (1991) first described Glut-1 DS in 2 children and subsequently reported 20 patients with various mutations. Symptoms present in the first year, with delayed motor and mental development, worsened by fasting, seizures refractory to antiepileptic drugs (AED) and aggravated by phenobarbital, and hypoglycorrhachia. A spinal tap and CSF glucose determination are important in a diagnostic workup of infants and young children with AED-resistant seizures, developmental delay, and ataxia. A decreased erythrocyte 3-OMG uptake and demonstration of heterozygous GLUT-1 mutations confirm the diagnosis. Barbiturates and methylxanthines, found to aggravate the seizures, should be avoided, and the ketogenic diet is used in treatment, at least in early childhood. Patients may continue to suffer from episodic limpness, ataxia, and confusion.

\title{
RESPIRATORY FAILURE IN ACID MALTASE DEFICIENCY
}

Sleep-disordered breathing (SDB) and respiratory failure (RF) were studied in 27 patients with juvenile and adult acid maltase deficiency (AMD) and compared with polysomnography outcomes at the University of Essen, Germany. Ventilatory restriction was present in $17 / 27$ patients, and inspiratory vital capacity correlated with peak inspiratory muscle pressure and gas exchange by day and night. Diaphragm weakness occurred in $13 / 27$, and was associated with SDB and RF. SDB was characterized by REM-sleep hypopneas and nocturnal hypoventilation. Treatment of RF or hypoventilation with noninvasive ventilation corrected daytime and nocturnal gas exchange. (Mellies $\mathrm{U}$, Ragette $\mathrm{R}$, Schwake C et al. Sleep-disordered breathing and respiratory failure in acid maltase deficiency. Neurology October (1 of 2) 2001;57:1290-1295). (Reprints: Dr Uwe Mellies, Department of Pneumology/Sleep Medicine, Ruhrlandklinik, Tuschener Weg 40, D45239 Essen, Germany).

COMMENT. Acid maltase deficiency (AMD), Type II glycogenosis or Pompe's disease, a rare hereditary myopathy, is an autosomal recessive glycogen storage disease that is complicated by heart, CNS and skeletal muscle dysfunction. It presents in childhood or in adults, and is slowly progressive, leading to respiratory failure and obstructive sleep apnea which may be fatal. In infants, acid maltase enzyme is deficient in lysosomes of heart, liver, and skeletal muscle, and glycogen is deposited in every tissue, including the CNS. First symptoms often appear by the second month and include difficulty in feeding, dyspnea, muscle weakness, and cardiac dysfunction, with marked cardiac enlargement. In late childhood and adult onset cases, organomegaly is absent, and muscle weakness is only slowly or nonprogressive, with involvement of lower limb proximal muscles. (Menkes JH, Textbook of Child Neurology, Lea \& Febiger, 1980). The above paper stresses the role of diaphragm weakness as the major cause of respiratory failure in juvenile and adult cases of $\mathrm{AMD}$, and the value of noninvasive ventilation in treatment of associated respiratory disorders.

\section{DEGENERATIVE DISORDERS}

\section{IRON METABOLISM AND HALLERVORDEN-SPATZ SYNDROME}

Hallervorden-Spatz syndrome (HSS) and iron metabolism are reviewed in a scientific workshop sponsored by the NIH and HSS Association in Bethesda, MD. First reported in 1922, the syndrome is now classified in 3 clinical types: 1) earlyonset childhood types, rapidly or slowly progressive; 2) late-onset, 10-18 years of age, slowly progressive; and 3) adult type, slowly progressive. Obligate 
characteristics are as follows: onset in the first two decades, progressive course, extrapyramidal signs (dystonia, rigidity, or choreoathetosis), MRI hypodensities corresponding with necrosis and iron deposits in basal ganglia, especially globus pallidus and substantia nigra, and the "eye-of-the-tiger" sign (higher signal intensity in centre and low signal on each side of globus pallidus).

Corrobarative signs (at least two) include: spasticity, intellectual deterioration, retinitis pigmentosa and/or optic atrophy, positive family history (autosomal recessive), and sea-blue histiocytes in bone marrow and/or abnormal cytosomes in circulating lymphocytes. Disorders to be excluded include: Wilson's disease, neuronal ceroid lipofuscinosis, juvenile Huntington's disease, GM1galactosidase or hexosminidase A deficiencies. The differential diagnosis also includes the HARP syndrome, a possible phenotype of HSS (hypobetalipoproteinemia, acanthocytosis, retinitis pigmentosa, and pallidal degeneration). The pathology. including iron metabolism and its accumulation in the basal ganglia, and symptomatic therapy are also discussed. (Swaiman KF. Hallervorden-Spatz syndrome. Pediatr Neurol August 2001;25:102-108). (Respond: Dr Swaiman, 1821 University Avenue W, N-188, St Paul, MN 55104).

COMMENT. The rare association of acanthocytosis and HSS has been reported by Swisher CN et al (1972), and the familial late infantile HSS has been described as a form of neuroacanthocytosis (Malandrini A et al, 1996). The above issue of Pediatric Neurology also includes reports on "Iron in the HSS," (Koeppen AH, Dickson AC, 2001), "Iron overload, oxidative stress, and axonal dystrophy in brain disorders," (Chiuch CC, 2001), "Systemic iron metabolism: a review and implications for brain iron metabolism," (Rouault TA, 2001); "Iron and iron management proteins in neurobiology," (Connor JR et al, 2001), and "Basal ganglia motor function in relation to Hallervorden-Spatz syndrome," (Mink JW, 2001). It is postulated that dystonia and other extrapyramidal signs of HSS are attributed to degeneration of basal ganglia output neurons. (See Progress in Pediatric Neurology III, PNB Publ, 1997;pp560-565, for further references to Harp and HSS syndromes).

\section{X-LINKED CHARCOT-MARIE-TOOTH DISEASE IN 93 PATIENTS}

The clinical, electrophysiological and genetic features of 93 patients (41 males, 52 females) from 37 unrelated families with X-linked dominant CharcotMarie-Tooth (CMTX) disease are reported from the Hopital de la Salpetriere, Paris, France. Age at onset was younger in males than females: $15.4+/-9.6$ yrs vs $18.7+1-$ $13.1 \mathrm{yrs}$. The respective age ranges of onset were 1-40 yrs in males and 1-56 yrs in females $(\mathrm{P}=0.22)$. Onset before age 10 years occurred in $27 \%$ of males and $15 \%$ of females. Disease duration at time of examination was shorter in males than females: $18.3+/-14.6$ yrs in males and $23.9+/-13.7$ yrs in females $(\mathrm{P}=0.11)$. Males were more severely affected than females and had higher functional disability scores; they had more frequent muscle weakness, amyotrophy, proprioception loss, upper limb areflexia, and pes cavus. Females were more frequently asymptomatic than males (29\% vs $11 \%)$. Motor nerve conduction velocities, distal motor latencies, and compound muscle action potentials were slower, longer and lower, respectively, in males compared to females. In molecular genetic testing, 27 different CX32 mutations and one entire deletion of the CX32 coding sequence were present in the 37 families. Patients with age at onset before 10 years presented nonfunctional mutations, and the type of mutation influences the phenotype. (Dubourg O, Tardieu S, Birouk $\mathrm{N}$ et al. Clinical, electrophysiological and molecular genetic characteristics of 93 patients with X-linked Charcot-MarieTooth disease. Brain October 2001;124:1958-1967). (Respond: Dr O Dubourg, Inserm 